International Journal of Mathematics and Systems Science (2018) Volume 1 doi:10.24294/ijmss.v1i4.898

\title{
A Comparative Study on the Ferrofluid Flow Models with Regards to the Behavior of A Ferrofluid Based Curved Rough Porous Circular Squeeze Film with Slip Velocity
}

\author{
Jimit R. Patel ${ }^{1}$, G. M. Deheri ${ }^{2}$ \\ ${ }^{1}$ Department of Mathematical sciences, P D Patel institute of Applied Sciences, Charusat, Changa, Gujarat, India. \\ ${ }^{2}$ Department of Mathematics, Sardar Patel University, Vallabh Vidyanagar, Anand, Gujarat, India.
}

\begin{abstract}
This investigation plans to introduce a correlation among all the three magnetic fluid flow models (Neuringer-Rosensweig's model, Shliomis's model, Jenkins's model) with regards to the conduct of a ferrofluid based curved rough porous circular squeeze film with slip velocity. The Beavers and Joseph's slip velocity has been invoked to assess the impact of slip velocity. Further, the stochastic model of Christensen and Tonder has been utilized to contemplate the impact of surface roughness. The load bearing capacity of the bearing system is found from the pressure distribution which is derived from the related stochastically averaged Reynolds type equation. The graphical portrayals guarantee that Shliomis model might be favored for preparation of the bearing system with improved life period. However, for lower to moderate values of slip Neuringer-Rosensweig model might be considered. Moreover, when the slip is at least the Jenkin's model might be deployed when the roughness is at reduced level.
\end{abstract}

Keywords: Circular Bearing, Ferrofluid, Roughness, Flow Models, Load Carrying Capacity

\section{Introduction}

The squeeze film, which has its own particular significance from quite a while, is utilized as a part of clutch plates, car transmissions and household apparatuses. Because of this, numerous examiners (Prakash and Vij (1973), Bhat (1978), Bhat and Deheri (1995), Deheri et al. (2005), Deheri and Patel (2011)) examined the ideal of squeeze film bearing.

It is realized that the nano particles are added to the base lubricants to upgrade the bearing effect in general. Ferrofluids, which are set up by scattering the magnetic particles in the fluid bearer, are a sort of smart materials. Because of their some vital physical and synthetic properties the magnetic fluids have been resorted to in various types of engineering and different fields applications, for example, vacuum sealing, magnetic reverberation, imaging, intelligent sensors, ink-jet printing, damper, cleaners etc.

A significant number of papers are accessible in the literature dealing with the discussions of various types of bearing systems adopting Neuringer-Rosensweig fluid flow model, for instance, Agrawal (1986), Shah and Bhat (2000), Shah and Bhat (2003), Nada and Osman (2007), Deheri and Abhangi (2011), Patel el al. (2012) Patel and Deheri (2016) and Patel el al. (2017). Jenkins (1972) changed the fluid flow model of Neuringer-Rosensweig (1964) by utilizing Maugin's alteration. It was observed that Neuringer-Rosensweig model changed the pressure while Jenkins flow model adjusted both the pressure and the viscosity of the ferrofluid. The steady-state effect of bearings with Jenkins model based magnetic fluids was investigated by Agrawal (1986), Ram and Verma (1999), Shah and Bhat (2002), Ahmad and Singh (2007), Patel and Deheri (2014) and Patel and Deheri (2015). It was shown that the bulk load carrying capacity of the bearing system expanded with increasing magnetization.

In 1972, Shliomis built up a ferrofluid flow model, in which the impacts of rotation of magnetic particles,

Copyright (C) 2018 Jimit R. Patel et al.

doi: 10.24294/ijmss.v1i4.898

EnPress Publisher LLC.This work is licensed under the Creative Commons Attribution-NonCommercial 4.0 International License (CC BY-NC 4.0). http://creativecommons.org/licenses/ by/4.0/ 
their magnetic moments and the volume concentration were inspected. From that point, numerous researchers (Kumar et al. (1992), Singh and Gupta (2012), Lin (2013), Patel and Deheri (2014)) examined the model of Shliomis to look at the execution of various bearing's systems. It was established that the unfriendly performance of transverse roughness could be overcome by the beneficial outcome of magnetization on account of negatively skewed roughness, appropriately picking the rotation parameter and the curvature parameters.

As usually known, surface roughness has been subjected to investigation in numerous examinations to enhance the tribological execution of the bearing system. Attributable to the significance of roughness, various investigators (Ting (1972), Praksh and Tiwari (1983), Guha (1993), Turaga et al. (1997), Gururajan and Prakash (2000), Gadelmawla et al. (2002), Sinha and Adamu (2009), Adamu and Sinha (2012) and Patel and Deheri $(2015,2016))$ supervised the effect of different kind of bearing systems by embracing the stochastic idea of Christensen and Tonder (1969a, 1969b, 1970).

Jao et al. (2016) proposed a hypothesis that incorporated the coupled impacts of surface roughness and anisotropic slips. It was found that the load proportion expanded as the dimensionless slip length diminished (with the exception of the instance of short bearing) or as the thinness proportion expanded. As of late, Patel and Deheri (2016) displayed the execution of an attractive liquid based parallel plate harsh slider holding for the correlation of all the three attractive liquid stream models (Neuringer-Rosensweig model, Shliomis model, and Jenkins demonstration).

In this way, it was thought appropriate to look into the joined impact of surface roughness and slip velocity on squeeze film attributes of circular plates bearing by considering the examination of three magnetic fluid flow models, in particular, Neuringer-Rosensweig model, Shliomis model and Jenkins model.

\section{Analysis}

Figure 1 contains the geometric configuration of the squeeze film circular bearing which has two circular plates, each of radiuses a. The upper curved plate approaches the lower one with normal uniform velocity $\dot{\mathrm{h}_{0}}$, where $\mathrm{h}_{0}$ is the central film thickness.

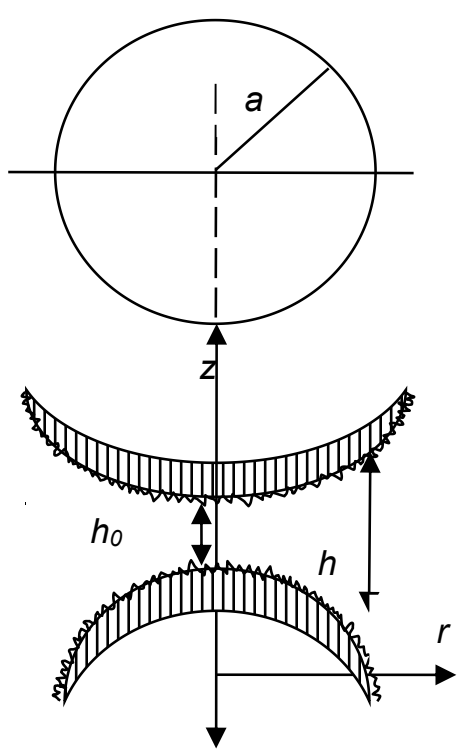

Figure 1; Physical configuration of the bearing system.

Bearing surfaces are presumed to be transversely rough. In view of Christensen and Tonder (1969a, 1969b, 1970), the thickness of the lubricant film takes the form

$$
\mathrm{h}=\overline{\mathrm{h}}+\mathrm{h}_{\mathrm{s}}
$$

where $\bar{h}$ denotes the mean film thickness and $\mathrm{h}_{\mathrm{s}}$ represents the deviation from the mean film thickness characterizing the random roughness of the bearing surfaces. $h_{s}$ is determined by the probability density function

$$
f\left(h_{s}\right)=\left\{\begin{array}{c}
\frac{35}{32 c}\left(1-\frac{h_{s}^{2}}{c^{2}}\right)^{3},-c \leq h_{s} \leq \mathrm{c} \\
0 \quad, \text { elsewhere }
\end{array}\right.
$$

c being the maximum deviation from the mean film thickness. The related facts mean $\alpha$, the standard deviation $\sigma$ and the parameter $\varepsilon$, which is the measure of symmetry of the random variable $h_{s}$ are culled from in Christensen and Tonder(1969a, 1969b, 1970).

A study of the discussions of Bhat (2003), Abhangi and Deheri (2011) and Patel and Deheri (2014, 2015), advances the opinion that the upper plate lying along the surface determined by the relation

$$
\mathrm{z}_{\mathrm{u}}=\mathrm{h}_{0} \exp \left(-\beta \mathrm{r}^{2}\right) ; 0 \leq \mathrm{r} \leq \mathrm{a}
$$

approaches, with normal velocity $\dot{h}_{0}$ to the lower plate lying along the surface given by

$$
\mathrm{z}_{\mathrm{l}}=\mathrm{h}_{0}\left[\sec \left(\gamma \mathrm{r}^{2}\right)-1\right] ; 0 \leq \mathrm{r} \leq \mathrm{a}
$$

where $\beta$ and $\gamma$ are the curvature parameters of the corresponding plates. The film thickness then, is defined by (Bhat (2003) and Patel and Deheri (2015, 2016)) 


$$
\mathrm{h}(\mathrm{r})=\mathrm{h}_{0}\left[\exp \left(-\beta \mathrm{r}^{2}\right)-\sec \left(\gamma \mathrm{r}^{2}\right)+1\right] ; 0 \leq \mathrm{r} \leq \mathrm{a}
$$

Neuringer and Rosensweig (1964) presented a simple flow model to define the steady flow of magnetic fluids in the presence of slowly changing external magnetic fields. The following equations characterize the model

$$
\begin{gathered}
\rho(\overline{\mathrm{q}} \nabla) \overline{\mathrm{q}}=-\nabla \mathrm{p}+\eta \nabla^{2} \overline{\mathrm{q}}+\mu_{0}(\overline{\mathrm{M}} \nabla) \overline{\mathrm{H}} \\
\nabla \overline{\mathrm{q}}=0 \\
\nabla \times \overline{\mathrm{H}}=0 \\
\overline{\mathrm{M}}=\bar{\mu} \overline{\mathrm{H}} \\
\nabla(\overline{\mathrm{H}}+\overline{\mathrm{M}})=0
\end{gathered}
$$

where $\rho$ denotes represents the fluid density, $\bar{q}$ represents the fluid velocity in the film region, $\overline{\mathrm{H}}$ denotes external magnetic field, $\bar{\mu}$ is magnetic susceptibility of the magnetic field, $p$ represents the film pressure, $\eta$ denotes the fluid viscosity and $\mu_{0}$ is the permeability of the free space. Further, details can be traced to Bhat (2003) and Prajapati (1995).

Using equations (4)-(5), equation (2) turns to

$$
\rho(\overline{\mathrm{q}} \nabla) \overline{\mathrm{q}}=-\nabla\left(\mathrm{p}-\frac{\mu_{0} \bar{\mu}}{2} \mathrm{M}^{2}\right)+\eta \nabla^{2} \overline{\mathrm{q}}
$$

The modified Reynolds equation governing the film pressure for Neuringer and Rosensweig model then, is found to be

$$
\frac{1}{\mathrm{r}} \frac{\mathrm{d}}{\mathrm{dr}}\left[\mathrm{h}^{3} \mathrm{r} \frac{\mathrm{d}}{\mathrm{dr}}\left(\mathrm{p}-\frac{\mu_{0} \bar{\mu}}{2} \mathrm{M}^{2}\right)\right]=12 \eta \dot{\mathrm{h}_{0}}
$$

Shliomis (1972) examined that magnetic particles of a magnetic fluid could relax in two ways when the applied magnetic field changed. One was due to the rotation of magnetic particles in the fluid and the other owing to rotation of the magnetic moment with in theparticles. In view of the deliberation of Bhat (2003) and Patel and Deheri (2014), the modified Reynolds type equation for Shliomis model takes the form

$$
\frac{1}{\mathrm{r}} \frac{\mathrm{d}}{\mathrm{dr}}\left(\mathrm{h}^{3} \mathrm{r} \frac{\mathrm{dp}}{\mathrm{dr}}\right)=12 \eta_{\mathrm{a}} \dot{\mathrm{h}}_{0}=12 \eta(1+\tau) \dot{\mathrm{h}_{0}}
$$

The specifics of the derivation of the equation is given in Bhat (2003) and Patel and Deheri $(2014,2015)$.

And Jenkins (1972) improved the approach of Neuringer-Rosensweig model developed a model to designate the flow of a ferrofluid. In view of Maugin's modification, equations for the model for steady states are (Jenkins (1972), Ram and Verma (1999), Patel and Deheri (2016)).

$$
\begin{aligned}
& \rho(\bar{q} \cdot \nabla) \bar{q}=-\nabla p+\eta \nabla^{2} \bar{q}+\mu_{0}(\bar{M} \cdot \nabla) \bar{H}+\frac{\rho A^{2}}{2} \nabla \\
& \times\left[\frac{\bar{M}}{M} \times\{(\nabla \times \bar{q}) \times \bar{M}\}\right]
\end{aligned}
$$

together with equations (4)-(7), A denotes a material constant. From equations (3) and (10) it is easily seen that Jenkins model is a generalization of NeuringerRosensweig model with an additional term

$$
\begin{aligned}
\frac{\rho J^{2}}{2} \nabla \times\left[\frac{\bar{M}}{\bar{M}} \times\{(\nabla\right. & \times \overline{\mathrm{q}}) \times \overline{\mathrm{M}}\}] \\
& =\frac{\rho \mathrm{A}^{2} \bar{\mu}}{2} \nabla \times\left[\frac{\overline{\mathrm{H}}}{\mathrm{H}} \times\{(\nabla \times \overline{\mathrm{q}}) \times \overline{\mathrm{H}}\}\right]
\end{aligned}
$$

Which improves the velocity of the fluid.

According to the discussions of Bhat (2003) and Patel and Deheri (2014), the changed Reynolds equation for Jenkins model assumes the form,

$\frac{1}{r} \frac{d}{d r}\left(\frac{h^{3}}{\left(1-\frac{\rho A^{2} \bar{\mu} M}{2 \eta}\right)} r \frac{d}{d r}\left(p-\frac{\mu_{0} \bar{\mu}}{2} M^{2}\right)\right)=12 \eta \dot{h_{0}}$

Considering the usual assumptions of hydrodynamic lubrication (Bhat (2003),Prajapati (1995), Deheri et al. (2005)) and the stochastic modelling of Christensen and Tonder (1969a, 1969b, 1970), the modified Reynolds' equation governing the pressure distribution turns out to be for Neuringer-Rosensweig model, Shliomis model and Jenkins model, respectively as,

$$
\begin{gathered}
\frac{1}{r} \frac{d}{d r}\left[g(h) r \frac{d}{d r}\left(p-\frac{\mu_{0} \bar{\mu}}{2} M^{2}\right)\right]=12 \eta \dot{h_{0}} \\
\frac{1}{r} \frac{d}{d r}\left(g(h) r \frac{d p}{d r}\right)=12 \eta(1+\tau) \dot{h_{0}}
\end{gathered}
$$

and

$$
\frac{1}{r} \frac{d}{d r}\left(\frac{g(h)}{\left(1-\frac{\rho A^{2} \bar{\mu} H}{2 \eta}\right)} r \frac{d}{d r}\left(p-\frac{\mu_{0} \bar{\mu}}{2} M^{2}\right)\right)=12 \eta \dot{h_{0}}
$$

where

$$
\begin{gathered}
g(h)=\left(h^{3}+3 h^{2} \alpha+3\left(\sigma^{2}+\alpha^{2}\right) h+3 \sigma^{2} \alpha+\alpha^{3}+\varepsilon\right. \\
\left.+12 \phi H_{0}\right)\left(\frac{4+s h}{2+s h}\right),
\end{gathered}
$$

$\phi$ is the permeability of the porous facing and $H_{0}$ is the thickness of the porous facing.

The following non dimensional quantities are considered for the study, 


$$
\begin{gathered}
\bar{h}=\frac{h}{h_{0}}, R=\frac{r}{a}, P=-\frac{h_{0}^{3} p}{\eta a^{2} \dot{h}_{0}}, B=\beta a^{2}, C=\gamma a^{2}, \\
\bar{\sigma}=\frac{\sigma}{h_{0}}, \bar{\alpha}=\frac{\alpha}{h_{0}}, \bar{\varepsilon}=\frac{\varepsilon}{h_{0}^{3}}, \\
M^{2}=k r^{2} \frac{(a-r)}{a}, \mu^{*}=-\frac{k \mu_{0} \bar{\mu} h_{0}^{3}}{\eta \dot{h}_{0}}, \bar{A}^{2}=\frac{\rho A^{2} \bar{\mu} \sqrt{k} a}{2 \eta}, \bar{s} \\
=s h_{0}, \bar{\Psi}=\frac{\phi H}{h_{0}^{3}}
\end{gathered}
$$

As usual the associated boundary conditions are

$$
P(1)=0,\left(\frac{d P}{d R}\right)_{R=0}=0
$$

Using the dimensionless quantities (16), the equations (13-15) convert respectively into,

$$
\begin{gathered}
\frac{1}{R} \frac{d}{d R}\left[g(\bar{h}) R \frac{d}{d R}\left(P-\frac{\mu^{*}}{2} R^{2}(1-R)\right)\right]=-12 \\
\frac{1}{R} \frac{d}{d R}\left(g(\bar{h}) R \frac{d P}{d R}\right)=-12(1+\tau) \\
\text { and } \\
\frac{1}{R} \frac{d}{d R}\left(\frac{g(\bar{h})}{\left(1-\bar{A}^{2} R \sqrt{1-R}\right)} R \frac{d}{d R}\left(P-\frac{\mu^{*}}{2} R^{2}(1-R)\right)\right) \\
=-12
\end{gathered}
$$

where

$$
\begin{gathered}
g(\bar{h})=\left(\bar{h}^{3}+3 \bar{h}^{2} \bar{\alpha}+3\left(\bar{\sigma}^{2}+\bar{\alpha}^{2}\right) \bar{h}+3 \bar{\sigma}^{2} \bar{\alpha}+\bar{\alpha}^{3}+\bar{\varepsilon}\right. \\
+12 \bar{\psi})\left(\frac{4+\bar{s} \bar{h}}{2+\bar{s} \bar{h}}\right)
\end{gathered}
$$

Solving equations (18-20) with the aid of the boundary conditions (16), the dimensionless pressure for Neuringer-Rosensweig model, Shliomis model and Jenkins model, respectively are determined by,

$$
\begin{aligned}
& P=\frac{\mu^{*}}{2} R^{2}(1-R)-6 \int_{1}^{R} \frac{R}{g(\bar{h})} d R \\
& P=-6(1+\tau) \int_{1}^{R} \frac{R}{g(\bar{h})} d R
\end{aligned}
$$

and

$$
P=\frac{\mu^{*}}{2} R^{2}(1-R)-6 \int_{1}^{R} \frac{R}{g(\bar{h})}\left(1-\bar{A}^{2} R \sqrt{1-R}\right) d R
$$

For all the three cases, the dimensionless load carrying capacity then turns out respectively as,

$$
\begin{gathered}
W=-\frac{h_{0}^{3}}{2 \pi \eta a^{4} \dot{h_{0}}} w=\int_{0}^{1} R P d R=\frac{\mu^{*}}{40}+3 \int_{0}^{1} \frac{R^{3}}{g(\bar{h})} d R \\
\begin{array}{c}
W=-\frac{h_{0}^{3}}{2 \pi \eta a^{4} \dot{h_{0}}} w=\int_{0}^{1} R P d R \\
=3(1+\tau) \int_{0}^{1} \frac{R^{3}}{g(\bar{h})} d R
\end{array}
\end{gathered}
$$

$$
\begin{gathered}
W=-\frac{h_{0}^{3}}{2 \pi \eta a^{4} \dot{h}_{0}} w=\int_{0}^{1} R P d R \\
=\frac{\mu^{*}}{40}+3 \int_{0}^{1} \frac{R^{3}}{g(\bar{h})}\left(1-\bar{A}^{2} R \sqrt{1-R}\right) d R
\end{gathered}
$$

\section{Results and discussions}

It is effortlessly observed that expression (24-26) decide the non dimensional load carrying capacity. It is noticed that the load carrying capacity gets augmented because of magnetization, when compared with the conventional oil based bearing system. It is entrenched reality that viscosity of the lubricant gets increment owing to magnetization, which adds to the expanded pressure bringing about raised load carrying capacity. Further, the way that the load carrying capacity upgrades because of magnetization can be seen through the associated equations (24-26) which are linear with respect to magnetization.

The variation of $W$ with respect to the magnetization exhibited in figures 2-7 underlines that an expansion in the magnetic outcomes with regards to the load carrying capacity, the most increment being registered on account of Shliomis model.

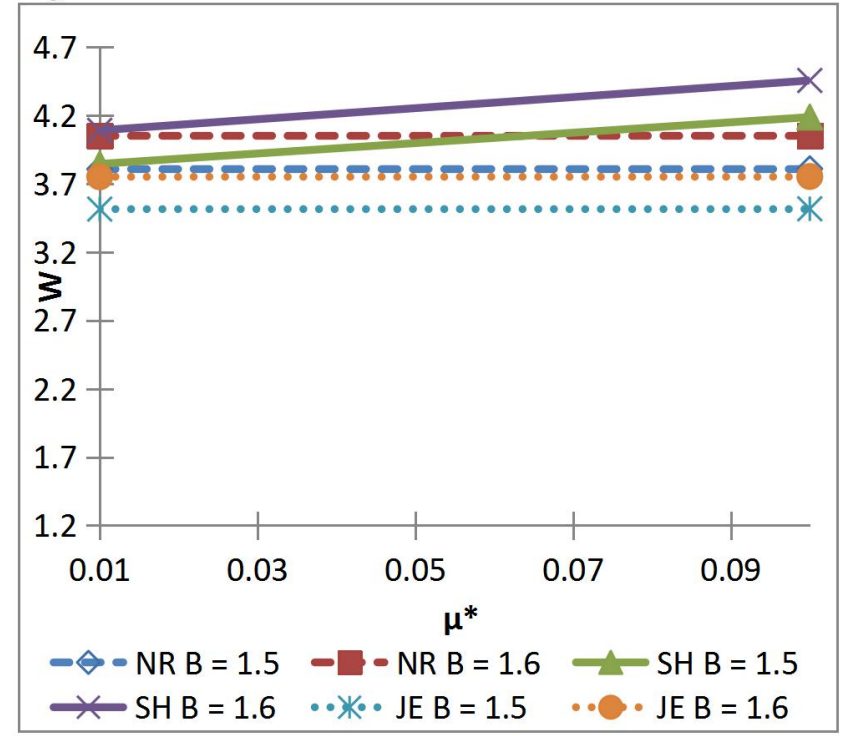

Figure 2; Variation of W with respect to $\mu^{*} / \tau$ andB.

and 


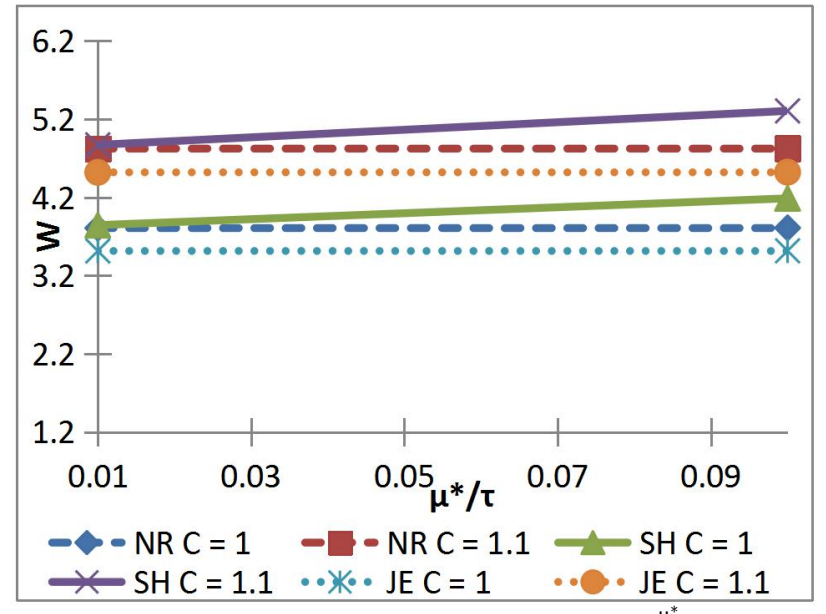

Figure 3; Variation of $\mathrm{W}$ with respect to $\frac{\mu^{*}}{\tau}$ and $\mathrm{C}$.

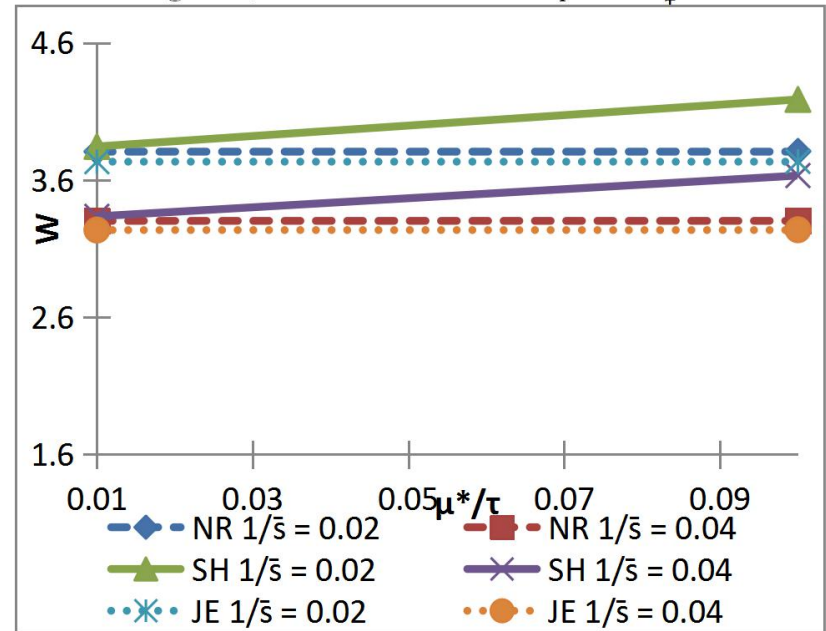

Figure 4; Variation of $W$ with respect to $\mu^{*} / \tau$ and $1 / \bar{s}$.

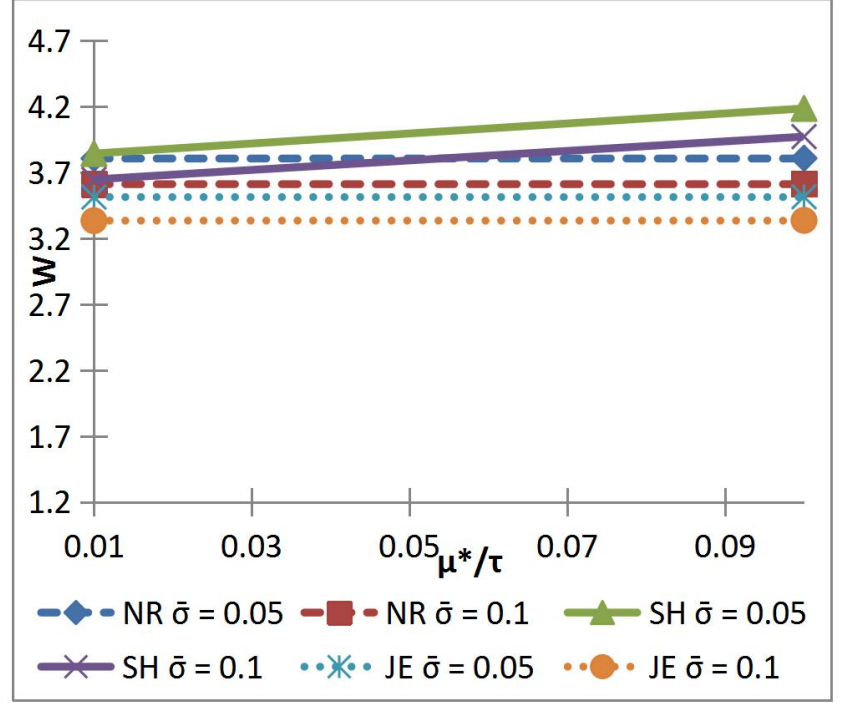

Figure 5; Variation of W with respect to $\mu^{*} / \tau$ and $\bar{\sigma}$.

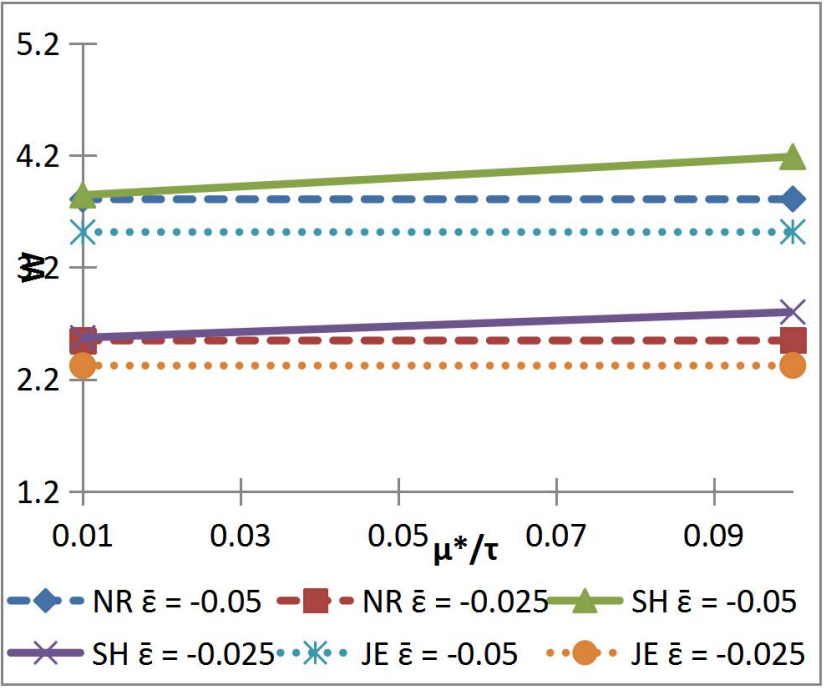

Figure 6; Variation of W with respect to $\mu^{*} / \tau$ and $\bar{\varepsilon}$.

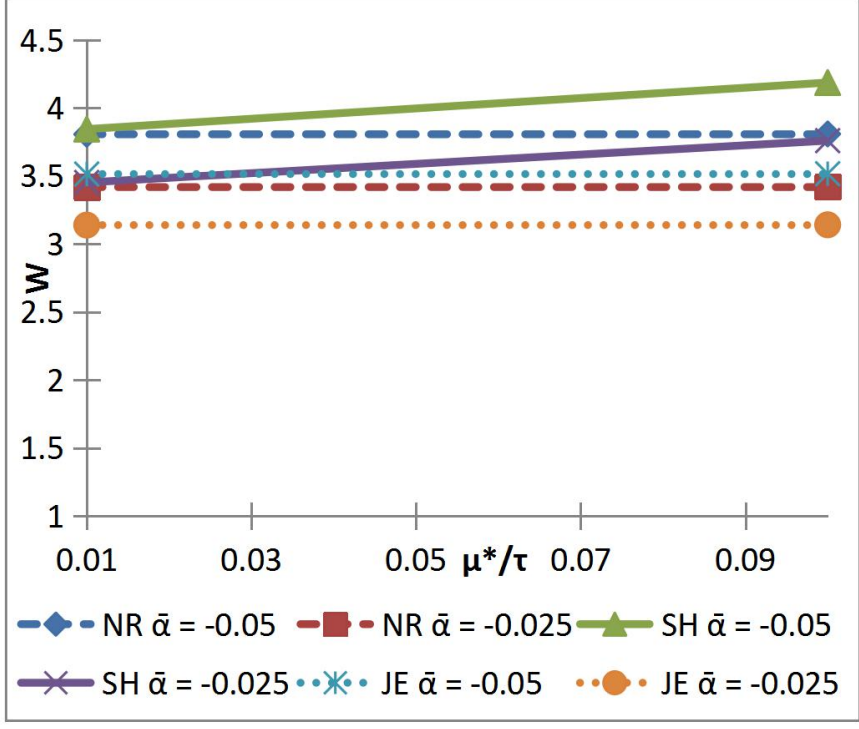

Figure 7; Variation of $W$ with respect to $\mu^{*} / \tau$ and $\bar{\alpha}$.

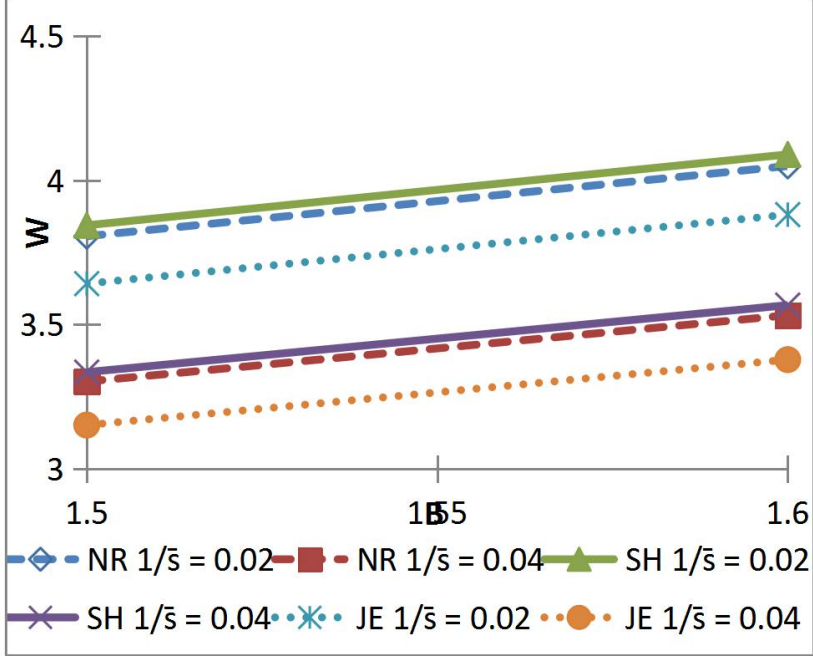

Figure 8; Variation of $W$ with respect to $B$ and $1 / \bar{s}$. 


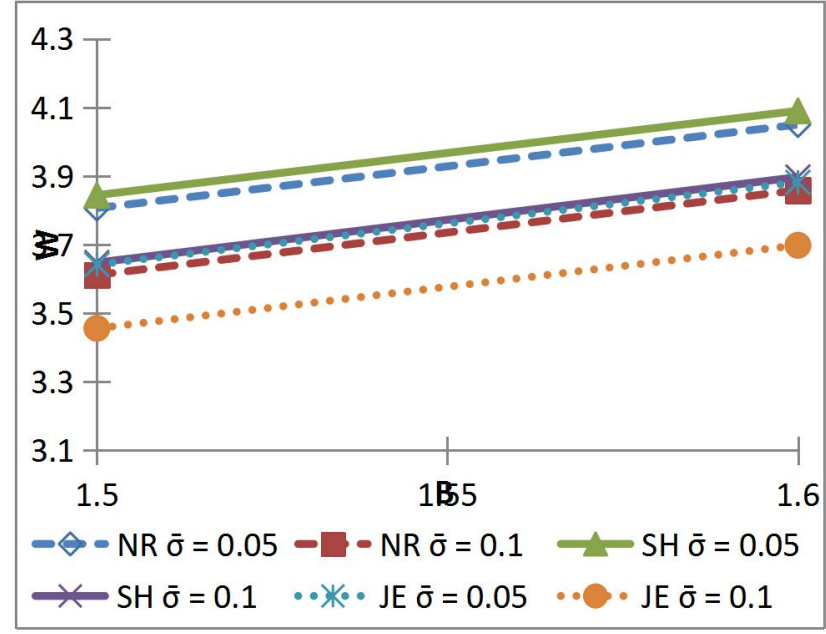

Figure 9; Variation of $\mathrm{W}$ with respect to Bandō.

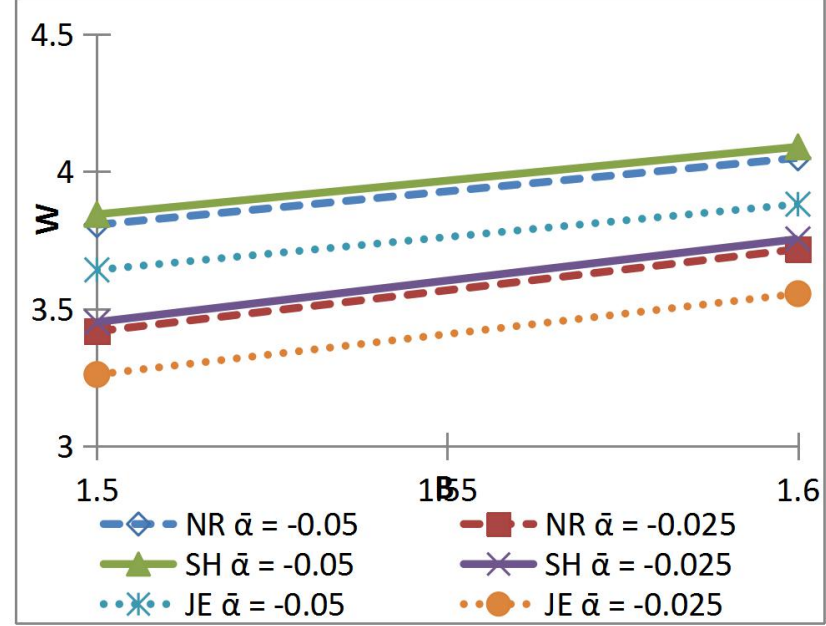

Figure 10; Variation of $W$ with respect to $B$ and $\bar{\alpha}$.

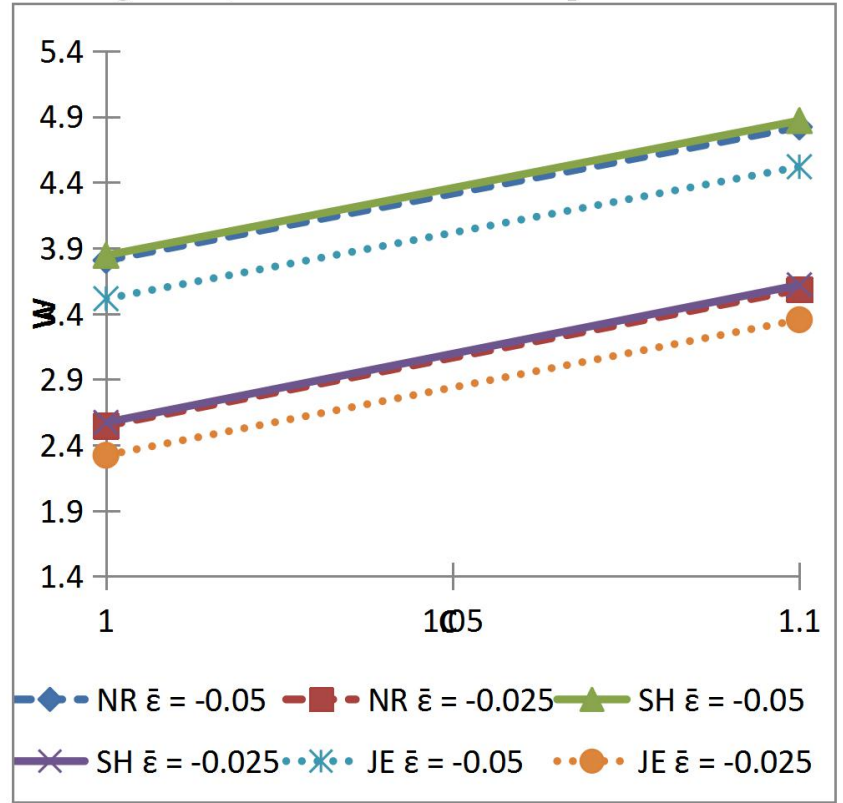

Figure 11; Variation of $W$ with respect to Cand $\bar{\varepsilon}$.

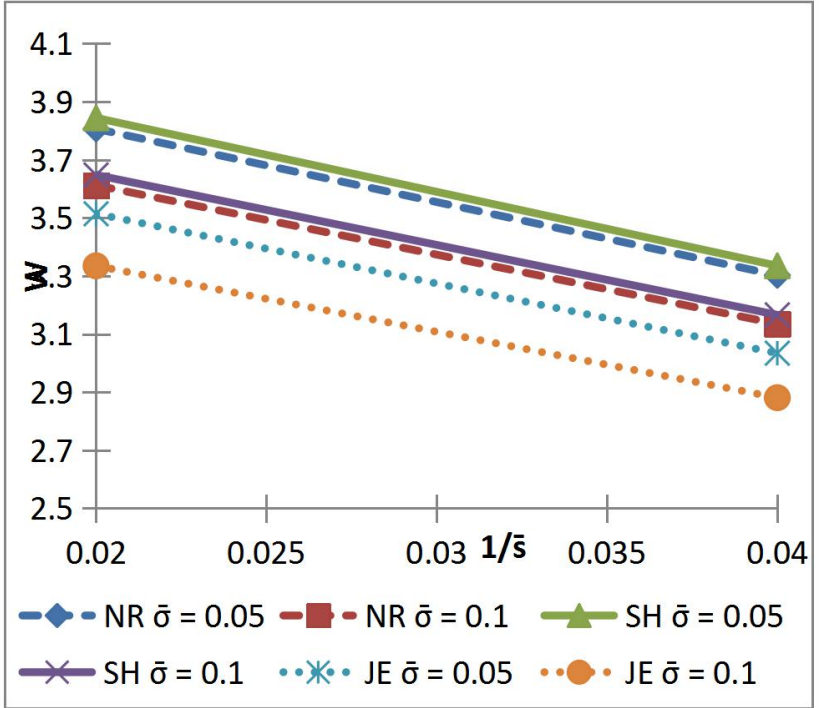

Figure 12; Variation of $W$ with respect to $1 / \bar{s}$ andō.

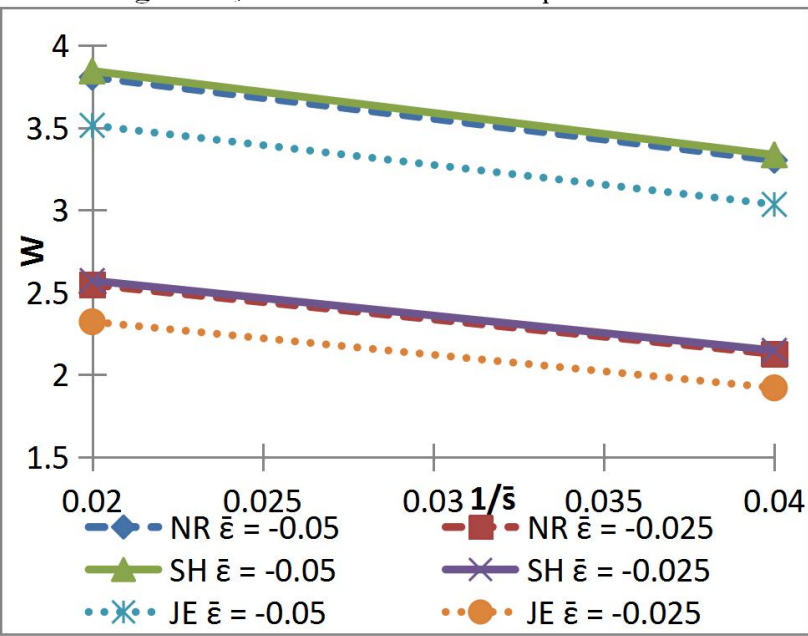

Figure 13; Variation of $W$ with respect to $1 / \bar{s}$ and $\bar{\varepsilon}$.

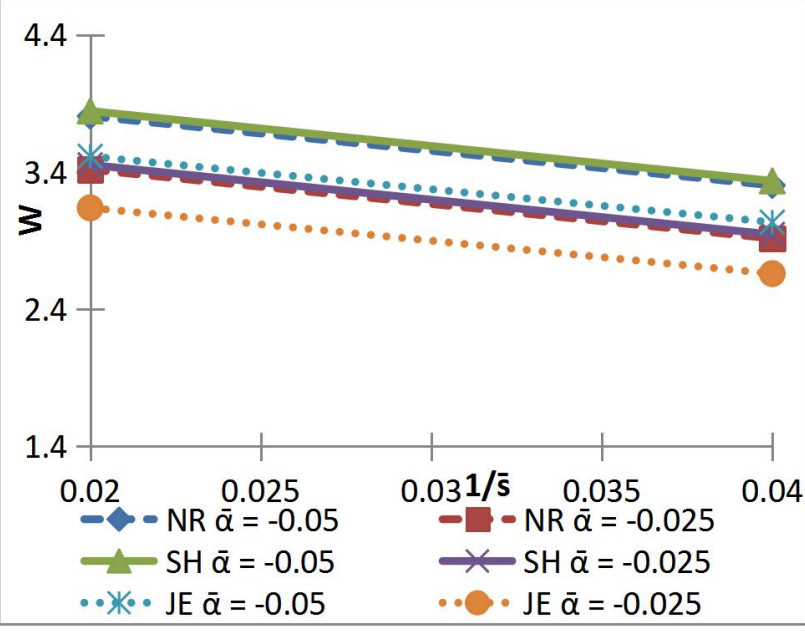

Figure 14; Variation of $W$ with respect to $1 / \overline{\operatorname{san}} d \bar{\alpha}$. 


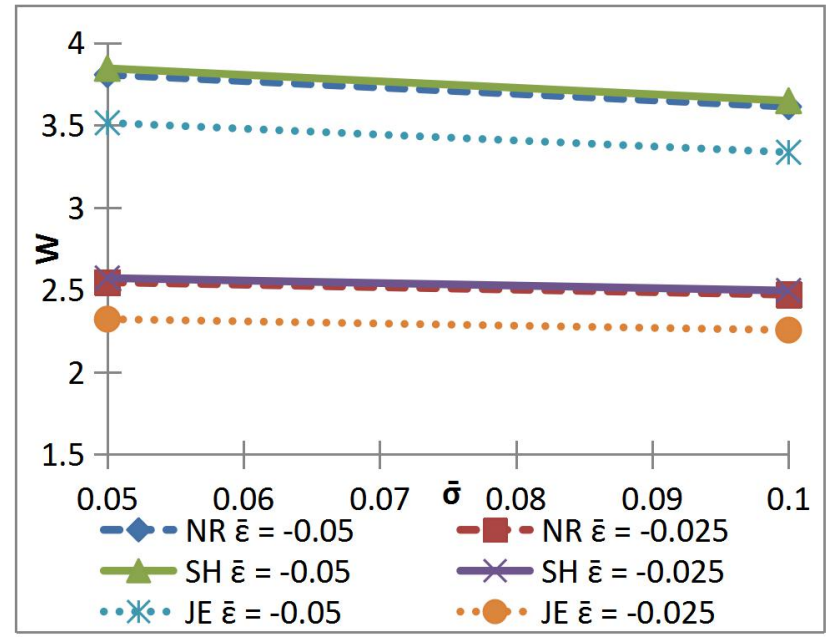

Figure 15; Variation of $W$ with respect to $\bar{\sigma}$ and $\bar{\varepsilon}$.

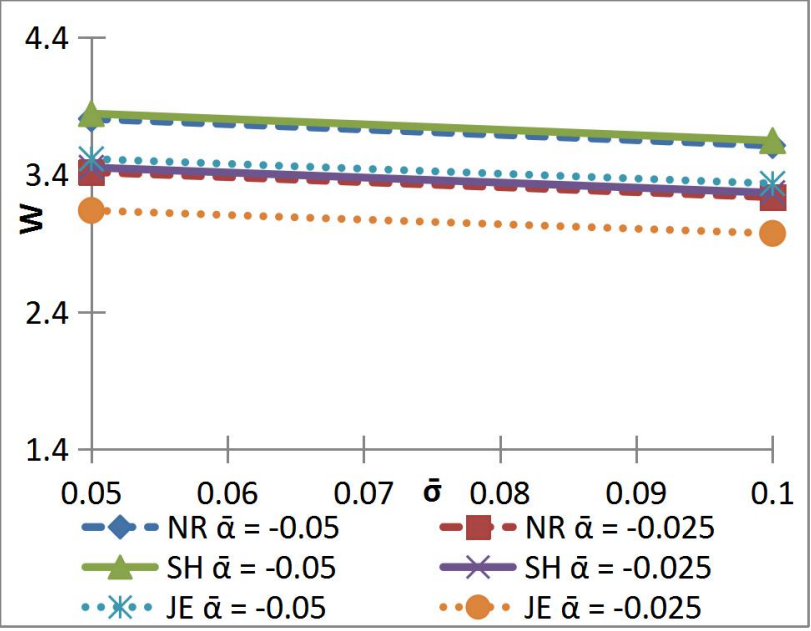

Figure 16; Variation of $W$ with respect to $\bar{\sigma}$ and $\bar{\alpha}$.

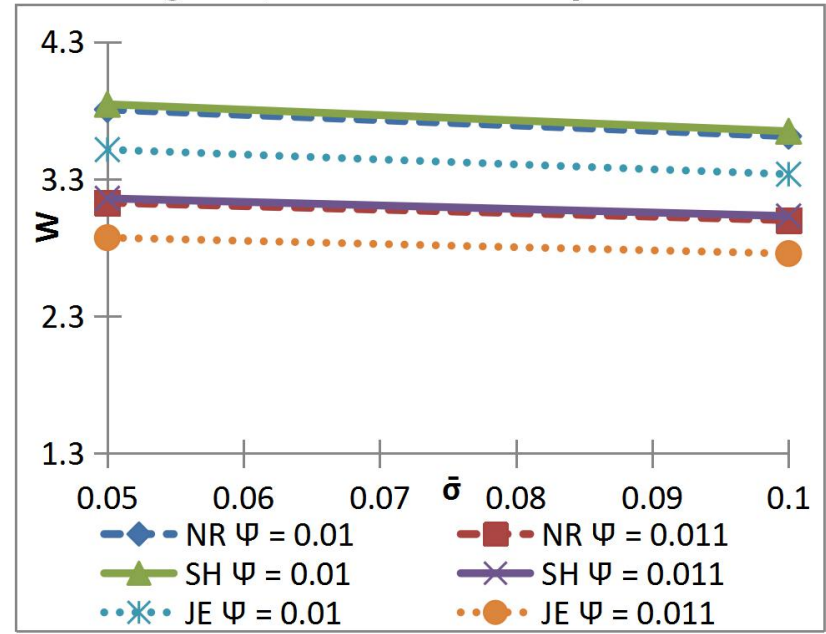

Figure 17; Variation of $W$ with respect to $\bar{\sigma}$ and $\bar{\psi}$.

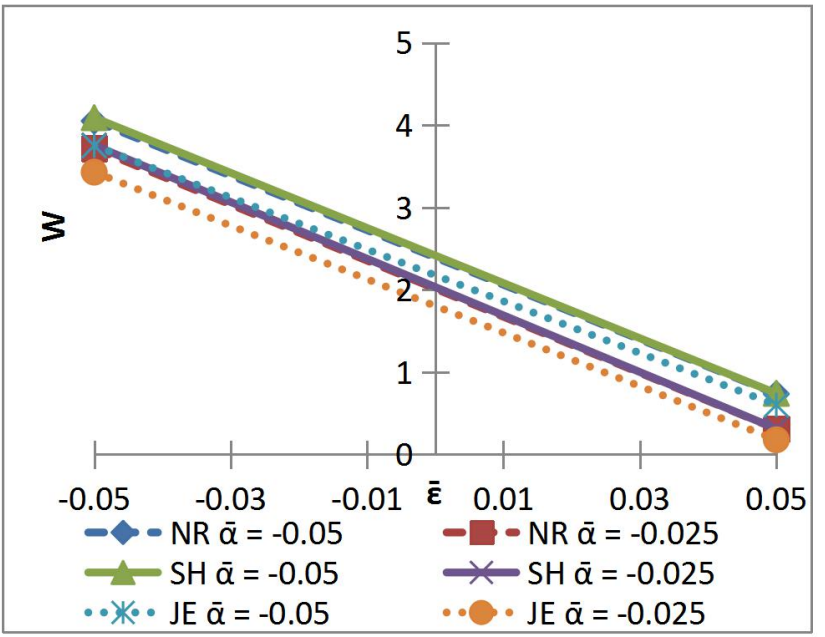

Figure 18; Variation of W with respect to $\bar{\varepsilon}$ and $\bar{\alpha}$.

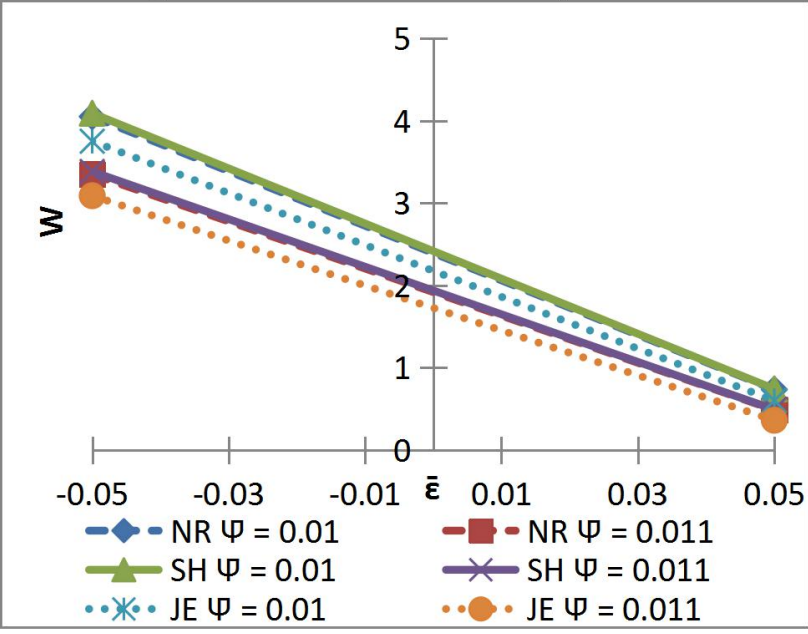

Figure 19; Variation of W with respect to $\bar{\varepsilon}$ and $\bar{\psi}$.

The consolidated impact of curvature parameters given in figures 8-11, recommends that the lower plate's shape parameter influences the most on account of Jenkin's model.

However, the slip effect experienced in figures 12-14 shows that the performance of slip parameter is quite more in the case of Jenkin's model.

The outcome of transverse surface roughness on $\mathrm{W}$ witnessed in figures 15-20 establish that the unfavorable effect of transverse surface roughness is enrolled to be more on account of Jenkin's model. In any case, for moderate to higher values of roughness parameter the Shliomis model puts back the Neuringer-Rosensweig model, in bringing down the impact of surface roughness.

As porosity tends to diminish the load carrying capacity the situation gets aggravated due to the slip effect.

A close take at the examination of the graphical 
portrayal has a tendency to put forth the accompanying,

- All the three models enhance the bearing performance when compared with conventional lubricant based bearing system. This is not out of the way because magnetization turns in an increase in the viscosity, leading to enhanced pressure.

- But the Shliomis model ends up being more favourable in comparison with the other two models with regards to roughness. Further, Neuringer-Rosensweig model and Jenkin's model vary a little when the consolidated effect of skewness and variance is considered.

- A key point to be seen is that the standard deviation brings down the load carrying capacity which is in contrast with the instance of parallel plate slider bearing without slip (Patel and Deheri (2016)).

-The combined impact of negatively skewed roughness and variance (- ve) may provide some measure of assistance to boost the performance of the bearing system for all the three models when the slip is at lower level.

-Exclusively, in the event that one considers the consolidated effect of roughness and slip, the Shliomis model surges ahead of the remaining two models.

- Up to certain level, the impact of standard deviation remains more prominent in Neuringer-Rosensweig model when contrasted with Jenkin's model.

-An examination of the figures displayed here enables us to infer that the load carrying capacity gets added at any rate by 2 to 3 percent when compared with the case of conventional fluid based curved rough porous circular squeeze film

-Besides, the Shliomis model ventures out in front of the other two models, in decreasing the unfavourable effect of porosity- slip combination.

\section{Conclusion}

The examination witnessed here discovers that the Neuringer-Rosensweig model might be conveyed to counter the effect of surface roughness when the slip effect is at reduced level. Be that as it may, for a bearing design with the long run the Shliomis model might be favored for moderate to higher loads, independent of the slip effect. For nominal roughness and moderate slip the performance of Neuringer-Rosensweig model and
Jenkins' model are almost identical. Also, the load upheld by the bearing system without flow is essentially higher on account of Shliomis model, which is unheard of in the case of conventional lubricant based bearing system. But this study simultaneously underlines that the roughness viewpoint is required to be dealt with while planning the bearing system regardless of the fact that Shliomis model has been utilized.

\section{References}

1. J. Prakash, S.K. Vij, Hydrodynamic lubrication of porous slider, J. Mech. Engg. Sci. 15(1973), 232-234

2. M.V. Bhat, Hydrodynamic lubrication of porous composite slider bearings, JPN Jour. of Applied Phys., 17(3) (1978), 479-481.

3. M.V. Bhat, G.M. Deheri, Porous slider bearing with squeeze film formed by a magnetic fluid, Pure and Applied mathematika sciences 39 (1-2) (1995) 39-43.

4. G.M. Deheri, P.I. Andharia, R.M. Patel, Transversely rough slider bearings with squeeze film formed by a magnetic fluid, Int. J. of Applied Mechanics and Engineering, 10(1)(2005) 53-76.

5. G.M. Deheri, J.R. Patel, Magnetic Fluid Based Squeeze Film in a Rough Porous Parallel Plate Slider Bearing, Annals of Faculty Engineering Hunedoara - International Journal of Engineering IX (3) (2011) 443-448,

6. R.C. Shah, M.V. Bhat, Squeeze film based on magnetic fluid in curved porous rotating circular plates, Journal of Magnetism and Magnetic Materials, 208(1) (2000) 115-119.

7. R.C. Shah and M.V. Bhat, Magnetic fluid based porous inclined slider bearing with velocity slip, Int. J. of Applied Mechanics and Engineering 18(2) (2003) 331-336.

8. G.S. Nada and T.A. Osman, Static performance of finite hydrodynamic journal bearings lubricated by magnetic fluids with couple stresses, Tribology Letters 27(3) (2007) 261-268.

9. G.M. Deheri, N.D. Abhangi, Numerical modelling of a magnetic fluid-based squeeze film between rotating transversely rough curved circular plates, International Journal of Computational Materials Science and Surface Engineering 4(3) (2011)185-204.

10. N.S. Patel, D.P. Vakharia, G.M. Deheri, A study on the performance of a magnetic-fluid-based hydrodynamic short journal bearing, ISRN Mechanical Engineering, 2012 (2012), Article ID 603460.

11. N.S. Patel, D.P. Vakharia, G.M. Deheri, H. C. Patel, Experimental performance analysis of ferrofluid based hydrodynamicjournal bearing with different combination of materials, Wear, Vol. 376-377, 2017, pp. 1877-1884. 
12. J.T. Jenkins, A theory of magnetic fluids, Archive for Rational Mechanics and Analysis 46(1972) 42-60.

13. J.L. Neuringer, R.E. Rosensweig, Magnetic Fluids, Magnetic Fluid, Physics of Fluids 7(12) (1964)1927.

14. V.K. Agrawal, Magnetic-fluid-based porous inclined slider bearing, Wear 107(2) (1986) 133-139.

15. P. Ram, P.D.S. Verma, Ferrofluid lubrication in porous inclined slider bearing, Indian Journal of Pure and Applied Mathematics 30(12) (1999)1273-1281.

16. R.C. Shah, M.V. Bhat, Ferrofluid lubrication in porous inclined slider bearing with velocity slip, International Journal of Mechanical Sciences 44(12) (2002) 2495-2502.

17. N. Ahmad, J.P. Singh, Magnetic fluid lubrication of porous pivoted slider bearing with slip velocity, Proceedings of the Institution of Mechanical Engineers Part J: Journal of Engineering Tribology 221(5)(2007) 609-613.

18. J.R. Patel, G. Deheri, Combined Effect of Surface Roughness and Slip Velocity on Jenkins Model Based Magnetic Squeeze Film in Curved Rough Circular Plates, International Journal of Computational Mathematics 2014 (2014) Article ID 367618.

19. J.R. Patel, G.M. Deheri, Jenkins model based ferrofluid lubrication of a curved rough annular squeeze film with slip velocity, Tribology in Industry 37(2) (2015) 129-141.

20. L.L. Ting, A Mathematical analog for determination of porous annular disk squeeze film behavior including the fluid inertia effect, J. Basic Engg.Trans.ASME, 94(1972) 417-421.

21. J. Prakash, K. Tiwari, Roughness effects in porous circular squeeze-plates with arbitrary wall thickness, J. Lubr. Tech. 105(1) (1983) 90-95.

22. S.K. Guha, Analysis of dynamic characteristics of hydrodynamic journal bearings with isotropic roughness effects, Wear 167 (2) (1993) 173-179.

23. Ram, Turaga, A.S. Sekhar, B.C. Majumdar, Stochastic FEM analysis of finite hydrodynamic bearings with rough surfaces, Tribology Transactions, 40 (4)( 1997) 605-612.

24. K. Gururajan, J. Prakash, Effect of surface roughness in a narrow porous journal bearing, $\mathrm{J}$. Tribology 122(2) (2000) 472-475.

25. E.S. Gadelmawla, M.M. Koura, T.M.A. Maksoud, I.M. Elewa, H.H. Sollman, Roughness parameters, Journal of materials processing Technology, 123 (1) (2002) 133-145.

26. P. Sinha, G. Adamu, THD analysis for slider bearing with roughness: special reference to load generation in parallel sliders, Acta Mech., 207 (2009), 11-27.
27. G. Adamu, P. Sinha, Thermal and Roughness Effects in a Tilted Pad Slider Bearing Considering Heat Conduction Through the Pad and Slider, Proceedings of the National Academy of Sciences, India Section A: Physical Sciences, 82(4) (2012) $323-333$.

28. H. Christensen, K.C. Tonder, Tribology of rough surfaces: stochastic models of hydrodynamic lubrication, SINTEF, 1969a , Report No.10/69-18.

29. H. Christensen, K.C. Tonder, Tribology of rough surfaces: parametric study and comparison of lubrication models, SINTEF, 1969b, Report No.22/69-18.

30. H. Christensen, K.C. Tonder, The hydrodynamic lubrication of rough bearing surfaces of finite width, ASME-ASLE Lubrication Conference, 1970, Paper no.70-lub-7.

31. M.I. Shliomis, Effective viscosity of magnetic suspensions, Sov. Physics JETP 34(6) (1972) 1291-1294.

32. D. Kumar, P. Sinha, P. Chandra, Ferrofluid squeeze film for spherical and comical bearings', Int. J. Engg. Sci. 30(5) (1992) 645-656.

33. U.P. Singh, R.S. Gupta, Dynamic performance characteristics of a curved slider bearing operating with ferrofluid, Advances in Tribology 2012 (2012), Article Id 278723.

34. J.R. Lin, Fluid inertia effects in ferrofluid squeeze film between a sphere and a plate, Applied Mathematical Modeling 37(7) (2013) 5528-5535.

35. J.R. Patel, G. Deheri, Shliomis model-based magnetic squeeze film in rotating rough curved circular plates: a comparison of two different porous structures, Int. J. of Computational Materials Sci. and Sur. Eng. 6(1) (2014) 29-49.

36. Hsiang-Chin Jao, Kuo-Ming Chang, Li-Ming Chu, Wang-Long Li, A Modified Average Reynolds Equation for Rough Bearings With Anisotropic Slip, Journal of Tribology, Vol. 138 , No. 1, pp. 011702-1-14, 2016.

37. J. R. Patel and G. M. Deheri, Performance of a Ferrofluid Based Rough Parallel Plate Slider Bearing: A Comparison of Three Magnetic Fluid Flow Models, Advances in Tribology, Volume 2016, Article ID 8197160, 2016.

38. M.V. Bhat, Lubrication with a Magnetic fluid, Team Spirit (India) Pvt. Ltd, 2003.

39. J. R. Patel and G. M. Deheri, Numerical modeling of Jenkins model based ferrofluid lubrication squeeze film performance in rough curved annular plates under the presence of slip velocity, Facta Universitatis, Ser. Math. Inform. Vol. 31, No 1 (2016), 11-31.

40. B.L. Prajapati, On Certain Theoretical Studies in Hydrodynamic and Electro-magneto hydrodynamic Lubrication, Ph.D. Thesis, S.P. University, Vallabh Vidya- Nagar, 1995. 